\title{
VARIACIÓN ALTITUDINAL Y DIVERSIDAD VEGETAL EN MATORRALES: SIERRA DE LOS FILABRES (ALMERÍA, ESPAÑA)
}

\author{
Julio PEÑAS, Javier CABELLO, Cecilio OYONARTE y Juan F. MOTA
}

\begin{abstract}
RESUMEN. Variación altitudinal y diversidad vegetal en matorrales: Sierra de Los Filabres (Almería, España). El objetivo principal de la investigación ha sido determinar la diversidad vegetal a lo largo del itinerario llevado a cabo en la cara sur de $S^{a}$ de Los Filabres situada en el sureste de la Península Ibérica, desde los $800 \mathrm{~m}$ de altitud hasta la cima. Las muestras fueron tomadas cada $100 \mathrm{~m}$ de desnivel. Además, se han tenido en cuenta magnitudes climáticas y bioclimáticas calculadas para cada estación, en relación con la diversidad registrada. Los aspectos corológicos se consideran también muy importantes para este análisis.
\end{abstract}

Palabras clave. Gradiente altitudinal, bioclimatología, riqueza específica, B-diversidad, similitud, corología, Andalucía, SE Península Ibérica.

ABSTRACT. Altitudinal variation and vegetational diversity in shrubs: Sierra de Los Filabres (Almería, Spain). The main objetives in this research is to determine vegetational diversity along the transect carried out on the south side of $S^{a}$ de Los Filabres, localiced in southeastern of Iberian Peninsula, from $800 \mathrm{~m}$ to the top. Samples were taken at $100 \mathrm{~m}$ intervals throughout the transect under study. Further, several climatic and bioclimatic parameters collected at every height station, have been brought into relationship with the available diversity record. Chorological aspects are very important too.

Keywords. Altitudinal gradient, bioclimatology, specific richness, ß-diversity, similarity, chorology, Andalusia, SE Iberian Peninsula.

\section{INTRODUCCIÓN Y OBJETIVOS}

La sierra de los Filabres es un gran macizo montañoso que se encuentra geográficamente ubicado en la zona central de la provincia de Almería, en el sureste de la Península Ibérica (fig. 1). Es una sierra fundamentalmente silícea
(Complejo Nevado-Filábride), con predominio de micaesquistos y cuarcitas, aunque también son extensas las áreas ocupadas por rocas carbonatadas. El notable gradiente altitudinal que existe, desde los $300 \mathrm{~m}$ a los $2168 \mathrm{~m}$, así como la gran variación latitudinal de oeste a este, le confiere unas especiales características

El presente trabajo ha sido financiado por la CICYT, bajo el proyecto de investigación NAT-89-0887-CO3-02. 


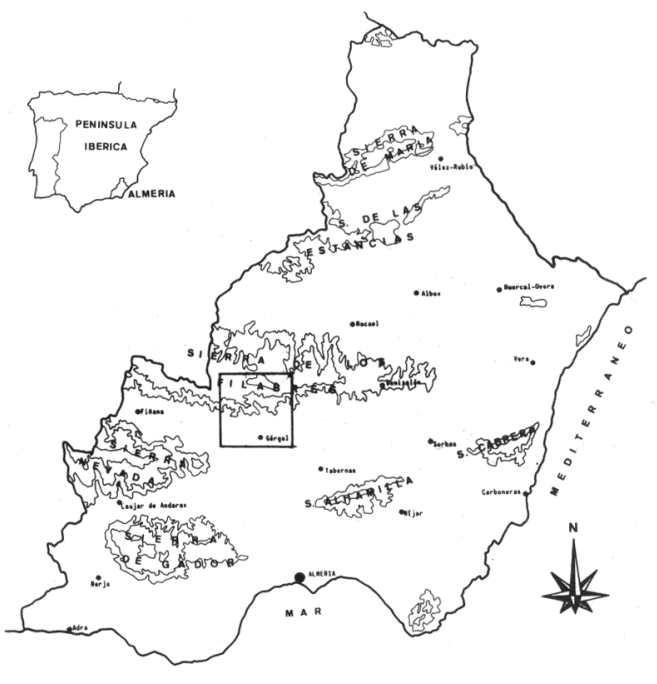

Fig. 1. Localización del área de estudio. Localization of study area.

climáticas y bioclimáticas, apareciendo, según las últimas propuestas de Rivas Martínez et al. (1993), dos tipos de zonobioclimas: mediterráneo xerofítico y mediterráneo mesofítico; cuatro termotipos: termomediterráneo, mesomediterráneo, supramediterráneo y oromediterráneo de forma topográfica; y tres ombrotipos: semiárido, seco y subhúmedo.

Desde el punto de vista biogeográfico la sierra de los Filabres presenta una interesante situación (Peñas et al., 1995), ya que en ella confluyen las provincias corológicas MurcianoAlmeriense (sector Almeriense) y Bética (sectores Nevadense y Guadiciano-Bacense). Por otro lado, la acción antrópica (centrada antíguamente en roturaciones para cultivos marginales y más recientemente en repoblaciones forestales mal planificadas) y la acción ganadera, han s ido muy agresivas con la vegetación, modelando en extremo el paisaje vegetal. Potencialmente deberían desarrollarse comunidades arbustivas (Bupleuro gibraltarici-Pistacietum lentisci) en las zonas basales, encinares béticos silicícolas (Adenocarpo decorticantis-Quercetum rotundifoliae) en las zonas intermedias y altas de la montaña, y formaciones aciculares de coníferas y piornos (Genisto versicolorisJuniperetum nanae) en las cacuminales; pero en su lugar dominan comunidades de matorrales seriales de muy diversa índole, siendo éstos a los que hemos restringido nuestro estudio.

Nuestro objetivo es relacionar la $\alpha$ diversidad (riqueza específica) y la $\beta$ diversidad (diferencia en diversidad de especies entre áreas o comunidades), de las fitocenosis de matorrales que se desarrollan en la $S^{a}$ de los Filabres, con el gradiente altitudinal en su cara sur, y por tanto con los cambios climáticos y bioclimáticos que se producen, como han realizado otros muchos autores en montañas de carácter mediterráneo (Ghanzanfar, 1991; Whittaker, 1960; Wilson \& Mohler, 1983; Wilson \& Shimida, 1984). Este último parámetro, la $ß$-diversidad ( $(\mathrm{w})$, resulta de especial interés ya que representa la rapidez de cambio de los hábitats que aparecen en el gradiente considerado (Kent \& Coker, 1992), en nuestro caso termoclimático y ombroclimático.

Para completar nuestro análisis, también se han usado otros parámetros relacionados con los puntos de muestreo, como el coeficiente de similitud y, a partir de éste, la tasa de renovación de especies.

\section{MATERIAL Y MÉTODOS}

Para la caracterización del clima se han realizado ecuaciones de correlación, establecidas a partir de 75 estaciones climatológicas del Instituto Nacional de Meteorología de España (18 termopluviométricas y 57 pluviométricas) localizadas tanto en la sierra de los Filabres como en áreas suficientemente cercanas y sin accidentes geográficos de importancia que perturbasen 
dichos datos. En las ecuaciones se relacionan la temperatura y la precipitación con la altitud y la longitud, calculándose estos dos parámetros climáticos (anual y mensualmente) para cada $100 \mathrm{~m}$ de desnivel altitudinal, desde $\operatorname{los} 800 \mathrm{~m}$ a $\operatorname{los} 2150 \mathrm{~m}$, coincidiendo con los puntos donde se muestreó la vegetación. Para el tratamiento estadístico de los datos climatológicos se ha utilizado el programa STATGRAPHIC.

Con los datos obtenidos se establecen una serie de índices que expresan la relación del clima con el crecimiento vegetal. A partir de los diagramas bioclimáticos propuestos por Montero de Burgos y Gonzalez Rebollar (1983), se obtienen la Intensidad Bioclimática Potencial (IBP), la Intensidad Bioclimática Real (IBR), en la que se distinguen las correspondientes al periodo cálido (IBRc) y al período frío (IBRf), la Intensidad Bioclimática Condicionada y la Intensidad Bioclimática Libre (IBL), representados igualmente para cada $100 \mathrm{~m}$. Para su cálculo se ha considerado una pérdida de agua por escorrentía constante en toda la catena del 10\%, dado la homogeneidad del relieve de la zona muestreada, asi como una capacidad de reserva de agua de $25 \mathrm{~mm}$ para los suelos situados por debajo de los $1500 \mathrm{~m}$ y de $50 \mathrm{~mm}$ por encima de esta altitud, valores que se consideran medios para los suelos del área de trabajo según el estudio edafológico sobre la Sierra de Filabres de Aguilar et al. (1987).

En el análisis de la bioclimatología local se han calculado el Indice de Termicidad Compensado (Itc), el Indice de Continentalidad (Ic) y el Indice Ombrotérmico (Io), propuestos por Rivas Martínez et al. (op. cit.), con los que se ha establecido la diagnosis bioclimática 0 piso bioclimático para cada estación de muestreo, que viene representado por el zonobioclima, el termotipo y el ombrotipo.

Los matorrales muestreados de la cara sur de la $S^{a}$ de Los Filabres corresponden a las etapas seriales de las siguentes sinasociaciones, desde las zonas basales hasta las cacuminales: - Serie termo-mesomediterránea cálida murciano-almeriense semiárido-seca inferior de la coscoja: Bupleuro gibraltarici-Pistacieto lentisci S.

- Serie meso-supramediterránea béticanevadense seca silicícola de encina faciación mesomediterránea: Adenocarpo decorticantisQuerceto rotundifoliae retametoso sphaerocarpae $S$.

- Serie meso-supramediterránea béticanevadense seca silicícola de encina faciación supramediterránea: Adenocarpo decorticantisQuerceto rotundifoliae quercetoso rotundifoliae $S$.

- Serie oromediterránea filábrico-nevadense silicícola del enebro rastrero: Genisto versicoloris-Junipereto nanae $S$.

Los muestreos de la vegetación se llevaron a cabo de forma cualitativa, registrando la presencia o ausencia de los taxones presentes en comunidades de matorral cada $100 \mathrm{~m}$ de desnivel en el transecto altitudinal (Wilson \& Shmida, op. cit.; Ghazanfar, op. cit.); fueron realizados por tres personas que recorrieron el territorio, paralelos a las curvas de nivel, durante 10 minutos. Se realizaron cada $100 \mathrm{~m}$ de altura, ya que en montañas mediterráneas no hay diferencias significativas en cambios de especies, respecto a muestreos cada $50 \mathrm{~m}$ (Auerbach \& Shmida, 1993).

Se consideraron un total de 78 taxones para dicha catena, estimando que la distribución de las especies que faltan en puntos intermedios es continúa entre la estación más baja y las más alta en que fueron detectados. Con estos datos se obtuvieron el número de especies en cada localidad muestral ( $\alpha$-diversidad), las ganadas y las perdidas en los muestreos sucesivos, a partir de estos la $\beta$-diversidad ( $B w$ ) propuesta por Whittaker (op. cit.) entre estaciones consecutivas y, el coeficiente de similitud entre el primer punto de muestreo (a los $800 \mathrm{~m}$.) y cada uno de los siguientes n-ésimos, calculado como sigue: 
(200 x $\mathrm{n}^{\circ}$ especies repetidas entre la estación 1 y la $\mathrm{n}$-ésima)

$\left(\mathrm{n}^{0}\right.$ especies de la estación $1+\mathrm{n}^{\circ}$ especies estación $n$-ésima)

Con la gráfica resultante de la distribución altitudinal de las especies a lo largo del gradiente, se ha realizado un análisis corológico de los taxones de cada punto de muestreo.

\section{RESULTADOS}

En la figura 2 se muestra el resultado de la distribución de las especies consideradas a lo largo del gradiente altitudinal, en él se detallan los puntos de muestreo ordenados de izquierda a derecha según disminuye la altura. Muy pocas especies se presentan en el rango completo muestreado (Artemisia glutinosa) o en la mayor parte (Helicrysum serotinum, Andryala ragusina, etc.); en la parte inferior de la figura aparecen las especies de más acentuada termofilia, como Asparagus horridus, Fumana thymifolia, Launaea fragilis, Hammada articulata, Carthamus arborescens, etc.

La tabla 1 expresa los valores de los datos climáticos y bioclimáticos calculados para las estaciones de muestreo; de acuerdo con la diagnosis bioclimática presentada pueden distinguirse seis zonas diferentes: mesomediterráneo xerofítico semiárido superior, mesomediterráneo mesofítico semiárido superior, mesomediterráneo mesofítico seco inferior, supramediterráneo mesofítico seco superior, supramediterráneo mesofítico subhúmedo inferior.

La tabla 2 especifica el número de taxones total muestreado, las especies ganadas y perdidas con respecto a la estación anterior y los valores de $B$ diversidad correspondientes. La fluctuación a lo largo del gradiente de estos dos parámetros se muestra en la figura 3. Por su parte, en la figura 4 podemos visualizar la variación del coeficiente de similitud.

El análisis corológico de los taxones registrados (ver anexo florístico) permite agruparlos en diez categorías biogeográficas: Murciano-Almerienses, Nevadenses, Béticos, Bético-Mogrebíes, Ibéricos, Ibero-Mogrebíes, Mediterráneo Occidentales, Mediterráneos, Mediterráneo-Asiático Occidentales, Otras distribuciones más amplias. Para su mejor comprensión fueron agrupados los taxones Béticos con los Bético-Mogrebíes, los Ibéricos con los Ibero-Mogrebíes, así como los Mediterráneos y Mediterráneo-Asiático Occidentales, mientras que el conjunto de especies englobado en "otras distribuciones» se desestimó por ser escasas y aportar poca luz al análisis. Por tanto, como se puede observar en la figura 5, centramos nuestro estudio en seis categorías especialmente significativas.

\section{DISCUSIÓN Y CONCLUSIONES}

Se han analizado cuatro aspectos a lo largo del gradiente altitudinal, riqueza específica, B-diversidad, similtud entre comunidades y espectro corológico.

Riqueza específica (figs. 2 y 3 ): La máxima riqueza específica de las comunidades vegetales aparece en las zonas de media montaña, fundamentalmente hasta los 1.400 $\mathrm{m}$, mientras que el mínimo se produce a partir de los 1700-1800 m, al igual que en otras montañas mediterráneo-orientales (Wilson \& Shmida, op. cit.), saharo-arábicas (Ghazanfar, $o p$. cit.) y $\mathrm{S}^{\text {a }}$ de Gádor en el sureste peninsular (Oyonarte et al., op. cit.). Por otra parte, los matorrales sobre rocas silíceas filábricas son menos ricos que los desarrollados sobre calizas (Oyonarte et al., op. cit.), algo que también ha sido puesto de manifiesto para las comunidades de pastizales de otras áreas (Grime, 1973 y 1979). 
Matorrales $S^{\mathbf{a}}$ de los Filabres

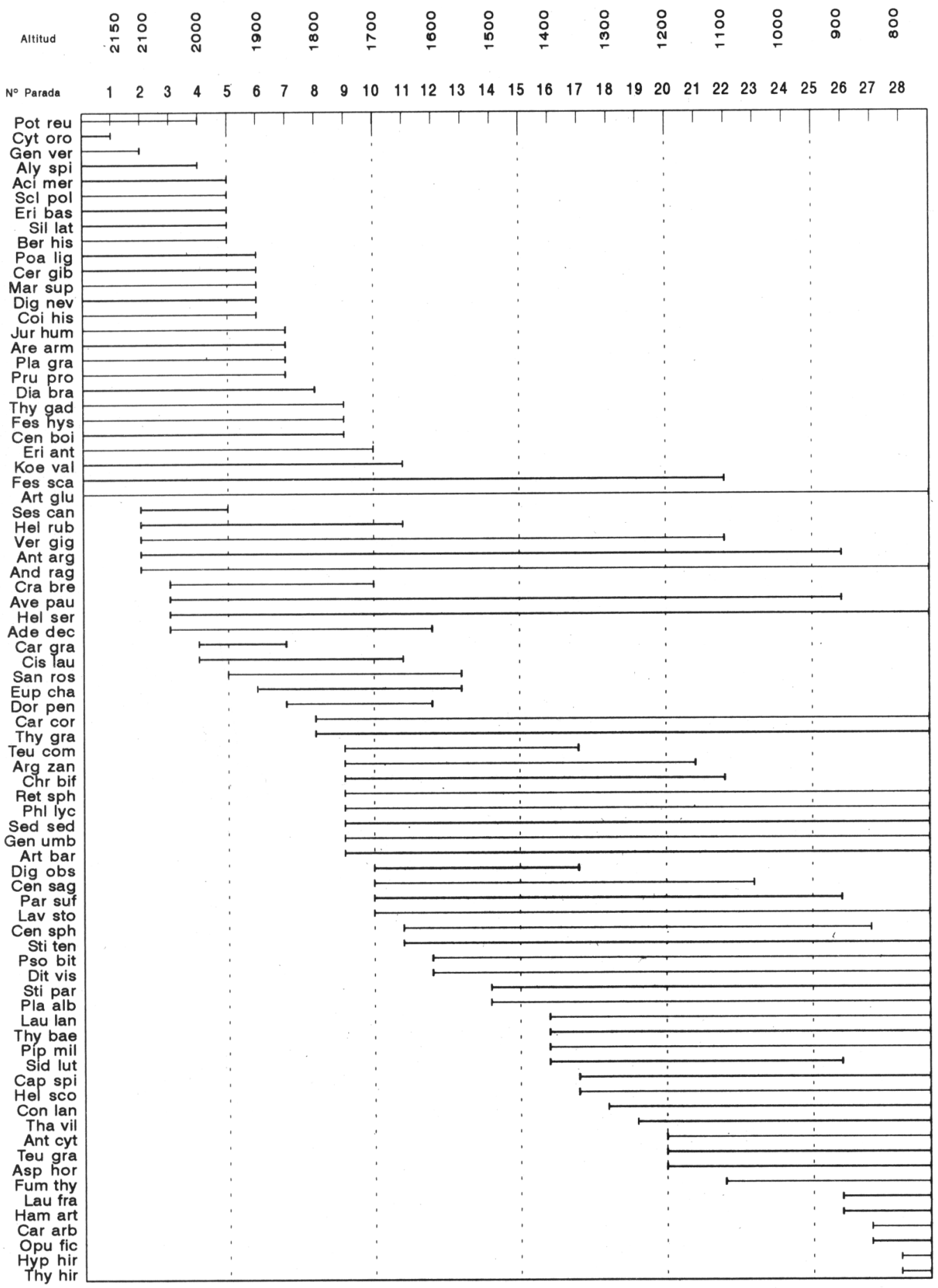

Figura 2. Distribución altitudinal de las especies. Cara Sur $S^{a}$ Filabres: Calar Alto-Gérgal. Altitudinal distribution of species. South side $S^{a}$ de los Filabres: Calar Alto-Gérgal. 


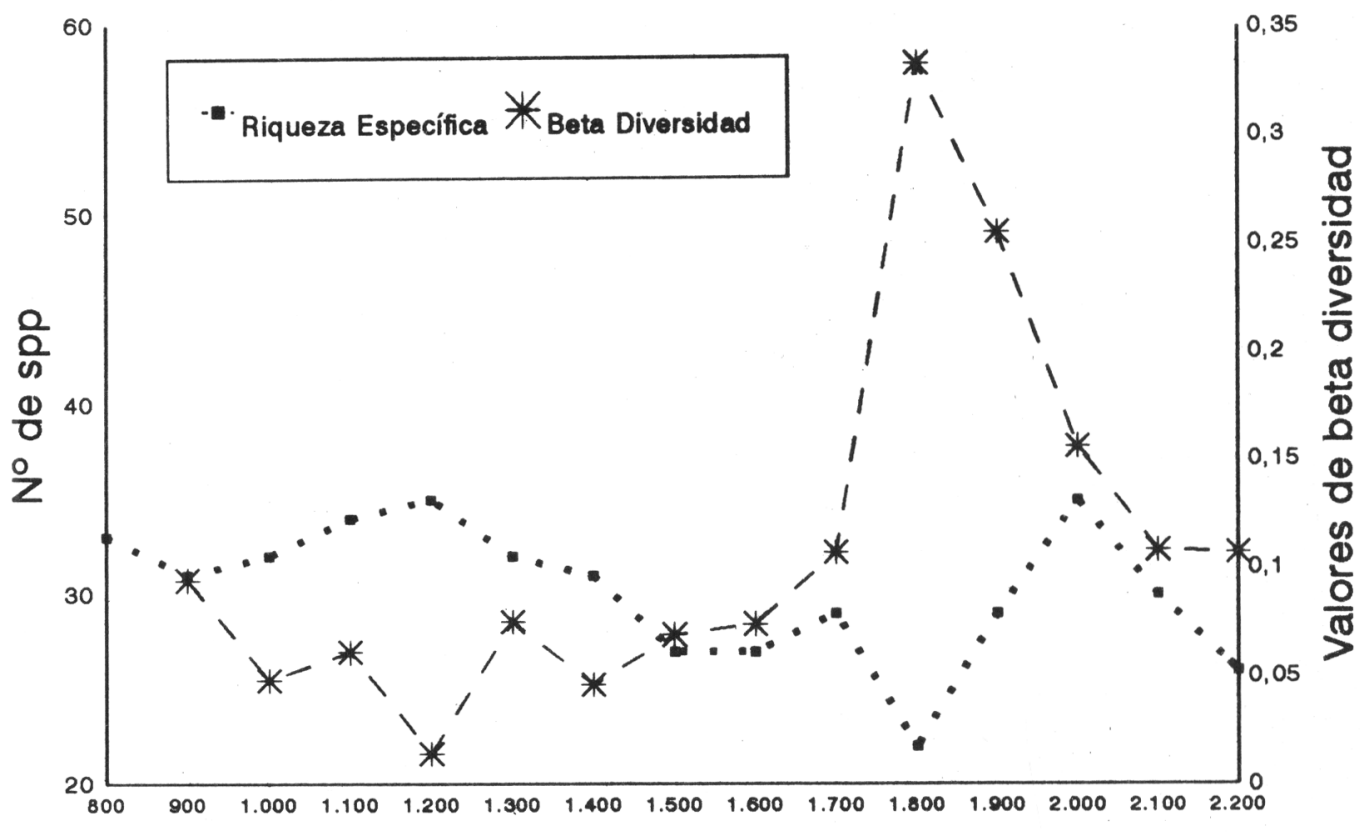

\section{Altitud}

Figura 3. Variación altitudinal de la Riqueza específica y de la Beta divesidad. Altitudinal variation of specific richness and $\beta$-diversity.

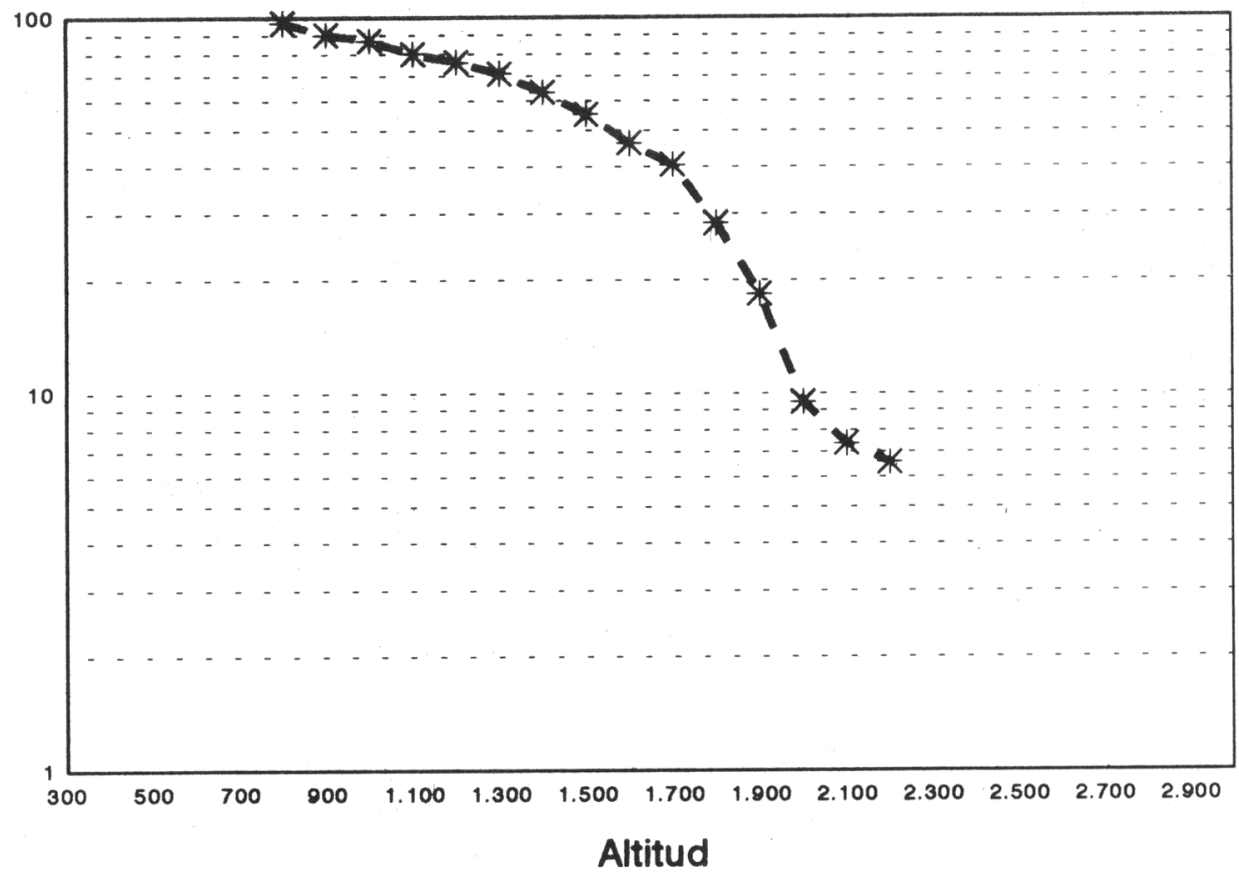

Figura 4. Coeficiente de similitud. Similarity coefficient. 
B-diversidad (Bw) (fig. 3 y tab. 1 y 2): La cara sur de los Filabres muestra un punto de inflexión hacia los $1800 \mathrm{~m}$, en el que este parámetro alcanza su máximo valor, lo que se interpreta como que la tasa de reemplazo de especies es elevada con respecto a los puntos altitudinales colindantes, esto sugiere un cambio brusco en los tipos de habitats. Se distinguen dos zonas que muestran una tendencia diferente de la $\beta$-diversidad; desde las partes basales hasta una altitud de aproximadamente 1700-1800 m va incrementando, mientras que decrece a partir de dicho punto.

En un trabajo anterior (Oyonarte et al., op. cit.) se propuso como causa del descenso de $B w$ la importancia que adquieren las condiciones microclimáticas imperantes en esas alturas. Analizando los datos de las sierras de los Filabres, también puede interpretarse este hecho en relación con la reducida superficie situada por encima de los $1800 \mathrm{~m}$ que existe en estas sierras de mediana altitud; esto determina la escasez de taxones orófilos (riqueza específica), lo que reduce las posibilidades de una fuerte competencia $y$ un intenso reemplazamiento de unas especies por otras (B-diversidad). Probablemente si se tratara de Sierra Nevada, que alcanza cotas superiores a los $3400 \mathrm{~m}$ y por tanto con una gran superficie por encima de los $1800 \mathrm{~m}$, el valor de Bdiversidad volvería a incrementarse, debido a la aparición de un gran número de endemismos nevadenses que sustituirían a los elementos anteriores en las zonas cacuminales. Esta última hipótesis se ve reforzada si tenemos en cuenta que este incremento ha sido puesto de manifiesto por otros autores (Wilson \& Shmida, $o p$. cit.) en cordilleras con mayor altitud.

Similitud y tasa de renovación (fig. 4): Se observa una variación lineal en el coeficiente de similitud a lo largo del gradiente aunque la curva incrementa su pendiente entre los $1700 \mathrm{y}$ $2150 \mathrm{~m}$, en coincidencia con el cambio drástico de condiciones ambientales que se producen a estas alturas y que ya veíamos reflejado en la B-diversidad. Esta observación ha permitido calcular la tasa de renovación, mediante una ecuación de regresión lineal, en unas 5,8 especies cada $100 \mathrm{~m}$, teniendo en cuenta tanto las que desaparecen como las que se incorporan.

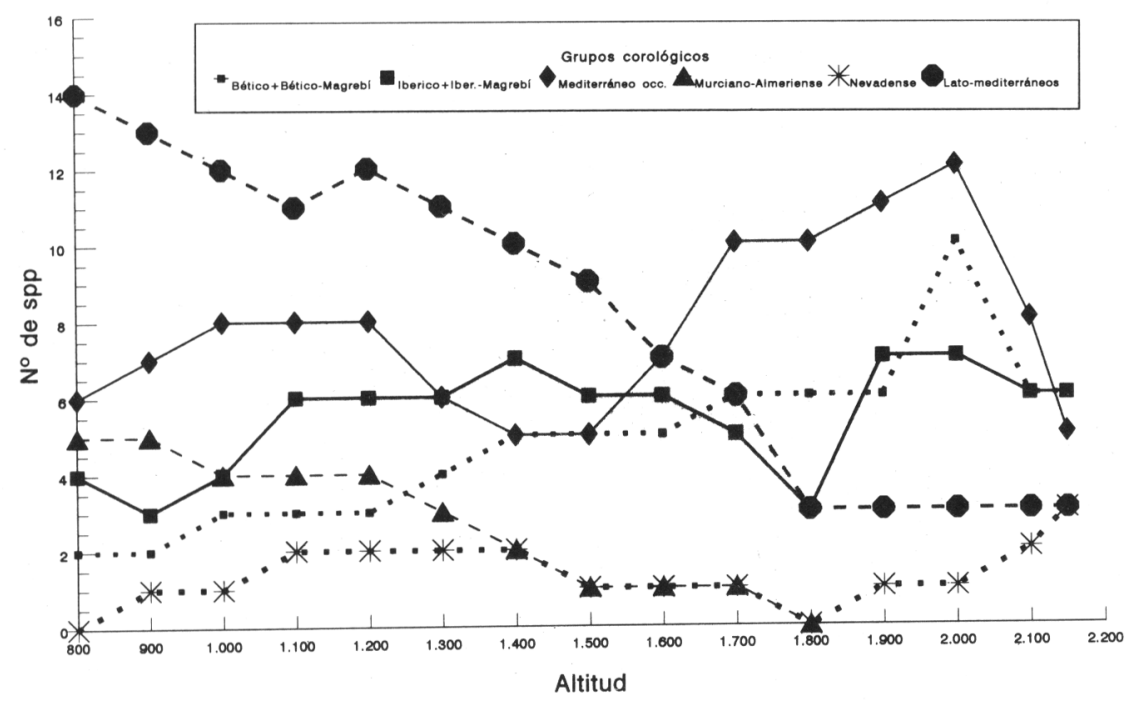

Figura 5. Espectro corológico a lo largo del gradiente. Chorological spectra along the gradient. 


\begin{tabular}{|c|c|c|c|c|c|c|c|c|c|c|c|c|}
\hline \multirow{2}{*}{$\begin{array}{l}\text { Altitud } \\
\text { (m) }\end{array}$} & \multirow{2}{*}{$\begin{array}{l}\text { Precip. } \\
\text { Med. Anual } \\
(\mathrm{mm})\end{array}$} & \multirow{2}{*}{$\begin{array}{c}\text { Temp. } \\
\text { Med. Anual } \\
\left({ }^{\circ} \mathrm{C}\right)\end{array}$} & \multirow{2}{*}{$\begin{array}{c}\text { IBS } \\
(1)\end{array}$} & \multirow{2}{*}{$\begin{array}{c}\text { IBP } \\
\text { (1) }\end{array}$} & \multicolumn{2}{|c|}{ IBR (1) } & \multirow{2}{*}{$\begin{array}{l}\text { IBL } \\
\text { (1) }\end{array}$} & \multirow{2}{*}{$\begin{array}{c}\text { IBC } \\
\text { (1) }\end{array}$} & \multirow{2}{*}{$\begin{array}{l}\text { Itc } \\
(2)\end{array}$} & \multirow{2}{*}{$\begin{array}{l}\text { Io } \\
\text { (2) }\end{array}$} & \multirow{2}{*}{$\begin{array}{l}\text { Ic } \\
\text { (2) }\end{array}$} & \multirow{2}{*}{$\begin{array}{l}\text { Diag. } \\
\text { Biocl. } \\
\text { (3) }\end{array}$} \\
\hline & & & & & (c) & (f) & & & & & & \\
\hline 800 & 319.5 & 15.0 & 1.82 & 17.9 & 2.44 & -- & 0.68 & 1.76 & 301 & 1.7 & 0 & 1 \\
\hline 900 & 331.4 & 14.4 & 1.7 & 19.8 & 2.07 & 0.1 & 0.56 & 1.51 & 283 & 1.9 & 0 & 1 \\
\hline 1000 & 343.5 & 13.9 & 1.58 & 15.7 & 1.8 & 0.34 & 0.46 & 1.34 & 268 & 2.1 & 0 & 2 \\
\hline 1100 & 355.4 & 13.3 & 1.47 & 14.7 & 1.59 & 0.7 & 0.44 & 1.15 & 250 & 2.2 & 0 & 3 \\
\hline 1200 & 367.3 & 12.8 & 1.38 & 13.7 & 1.33 & 1.06 & 0.37 & 0.96 & 233 & 2.4 & 0 & 3 \\
\hline 1300 & 379.1 & 12.2 & 1.31 & 12.9 & 1.13 & 1.52 & 0.32 & 0.81 & 216 & 2.6 & 0 & 3 \\
\hline 1400 & 391.1 & 11.7 & 1.23 & 12.1 & 0.97 & 2.04 & 0.28 & 0.7 & 201 & 2.8 & 1 & 4 \\
\hline 1500 & 403.3 & 11.1 & 1.16 & 11.4 & 0.91 & 2.66 & 0.24 & 0.68 & 186 & 3.0 & 4 & 4 \\
\hline 1600 & 415.1 & 10.6 & 1.09 & 10.7 & 0.81 & 3.3 & 0.19 & 0.62 & 172 & 3.3 & 6 & 4 \\
\hline 1700 & 427.2 & 10 & 1.05 & 10.2 & 0.79 & 4.04 & 0.25 & 0.54 & 157 & 3.6 & 9 & 5 \\
\hline 1800 & 439.1 & 9.5 & 1.01 & 9.6 & 0.76 & 4.8 & 0.3 & 0.46 & 143 & 3.8 & 11 & 5 \\
\hline 1900 & 451 & 8.9 & 0.97 & 9.0 & 0.68 & 5.52 & 0.33 & 0.35 & 127 & 4.2 & 13 & 5 \\
\hline 2000 & 463 & 8.4 & 0.92 & 8.4 & 0.58 & 6.26 & 0.33 & 0.25 & 113 & 4.6 & 15 & 5 \\
\hline 2100 & 475 & 7.8 & 0.89 & 7.9 & 0.52 & 7.02 & 0.31 & 0.2 & 98 & 5.1 & 18 & 6 \\
\hline 2150 & 481.1 & 7.6 & 0.87 & 7.7 & 0.5 & 7.54 & 0.29 & 0.21 & 91 & 4.8 & 19 & 6 \\
\hline
\end{tabular}

Tabla 1. Valores de las variables climáticas y bioclimáticas en cada estación de muestreo.(1) Índices Bioclimáticos. IBS: Intensidad Bioclimática Seca; IBP: Intensidad Bioclimática Potencial; IBR: Intensidad Bioclimática Real, (c) periodo cálido y (f) periodo frío; IBC: Intensidad Bioclimática Condicionada; IBL: Intensidad Bioclimática Libre. (2) Itc: Índice de Termicidad Compensado; Io: Índice Ombrotérmico; Ic: Índice de Continentalidad. (3) Diagnosis Bioclimática. 1: Mesomediterráneo Xerofítico Semiárido superior; 2: Mesomediterráneo Mesofítico Semiárido superior; 3: Mesomediterráneo Mesofítico Seco inferior; 4: Supramediterráneo Mesofítico Seco inferior; 5: Supramediterráneo Mesofítico Seco superior; 6: Supramediterráneo Mesofítico Subhúmedo inferior. Values of the climatic and bioclimatic variables in every samples station.

Espectro corológico (fig. 5): En la zona basal dominan elementos mediterráneos de área amplia (circunmediterráneos y mediterráneoasiático occidentales) hasta los 1600-1700 m, en donde comienzan a desaparecer, dejando paso a taxones de distribución más restringida; en este último grupo destacan por su elevado número los mediterráneo occidentales, seguidos del conjunto de bético-mogrebíes, béticos e ibéricos. Por otro lado, como se esperaba, los elementos endémicos de la Provincia Murciano-Almeriense presentan un gran protagonismo en las partes basales, pertenecientes biogeográficamente a esta unidad; son progresivamente sustituidos, a partir de los 1100-1200 m, por el conjunto de taxones propios de la provincia Bética cuando entramos en el dominio de ésta. En cuanto a los 


\begin{tabular}{lrrrrrrrrrrrrrrr}
\hline ALTITUD & 800 & 900 & 1000 & 1100 & 1200 & 1300 & 1400 & 1500 & 1600 & 1700 & 1800 & 1900 & 2000 & 2100 & 2150 \\
\hline RIQ. ESPECIFICA & 33 & 31 & 32 & 34 & 35 & 32 & 31 & 27 & 27 & 29 & 22 & 29 & 35 & 30 & 26 \\
ESP. GANADAS & - & 2 & 2 & 3 & 1 & 1 & 2 & 0 & 2 & 4 & 5 & 10 & 8 & 1 & 1 \\
ESP. PERDIDAS & - & 4 & 3 & 1 & 0 & 4 & 3 & 4 & 2 & 4 & 12 & 3 & 2 & 5 & 5 \\
B-DIVERSIDAD & -.094 & .048 & .061 & .014 & .075 & .046 & .069 & .074 & .107 & .333 & .255 & .156 & .108 & .107
\end{tabular}

Tabla 2.- Número total de taxones en cada estación de muestreo, especies ganadas y perdidas respecto a la estación anterior, y $B$-diversidad. Total number of taxa in every samples station, gained and lost species respect the anterior samples station, and $\beta$-diversity.

elementos florísticos nevadenses, destaca su escasez a pesar de que todo el territorio bético de los Filabres pertenece a este sector; la falta de más altura en esta sierra, como hemos indicado, hace imposible el establecimiento de más endemismos nevadenses.

Considerando en conjunto el espectro corológico y la $B$-diversidad, las mayores variaciones de esta última coinciden de manera muy estrecha con la importante sustitución de los elementos de origen mediterráneo amplio por los mediterráneo occidentales y béticos, $o$ lo que es lo mismo, el reemplazamiento de taxones de área amplia por otros de área más restringida, ya que al ascender en altitud mayor es el aislamiento fitogeográfico.

De la comparación entre el rango altitudinal de las especies, que refleja la figura 2, y los valores bioclimáticos que aparecen en la tabla 1 , se pueden establecer relaciones que expliquen el comportamiento ecológico de los taxones, pero la distribución de las especies y de las comunidades ne depende exclusivamente de los parámetros climáticos y bioclimáticos que varían con la altitud, sino también de otros aspectos relacionados con el suelo, interacciones biológicas, etc.

\section{ANEXO}

Apéndice florístico en el cual se abrevian los taxones infraespecíficos.
Aci mer (Acinos meridionalis); Ade dec (Adenocarpus decorticans); Aly spi (Alyssum spinosum); And rag (Andryala ragusina); Ant arg (Anthyllis argyrophylla); Ant cyt (Anthyllis cytisoides); Are arm (Arenaria armerina); Arg zan (Argyrolobium zanonii); Art bar (Artemisia barrelieri); Art glu (Artemisia glutinosa); Asp hor (Asparagus horridus); Ave pau (Avenula pauneroi); Ber his (Berberis hispanica); Cap spi (Capparis spinosa); Car gra (Carduus granatensis); Car cor (Carlyna corymbosa); Car arb (Carthamus arborescens); Cen sag (Centaurea sagredoi); Cen sph (Centaurea sphaerocephala); Cen boi (Centaurea boissieri); Cer gib (Cerastium gibraltaricum); Cis lau (Cistus laurifolius); Coi his (Coincya hispida); Con lan (Convolvulus lanuginosus); Cra bre (Crataegus brevispina); $\mathrm{Cyt}$ oro (Cytisus oromediterraneus); $\mathrm{Chr}$ bif (Chronanthus biflorus); Dia bra (Dianthus brachyanthus); Dig nev (Digitalis nevedensis); Dig obs (Digitalis obscura); Dit vis (Dittrichia viscosa); Dor pen (Dorycnium pentaphyllum); Eri ant (Erinacea anthyllis); Eri bas (Erisimum bastetanum); Eup cha (Euphorbia characias); Fes hys (Festuca hystrix); Fes sca (Festuca scariosa); Fum thy (Fumana thymifolia); Gen umb (Genista umbellata); Gen ver (Genista versicolor); Ham art (Hammada articulata); Hel sco (Helianthemum scopulorum); Hel ser (Helichrysum serotinum); Hel rub (Helianthemum rubellum); Hyp hir (Hyparrhenia hirta); Jur hum (Jurinea humilis); Koe val (Koeleria vallesiana); Lau lan (Launaea lanifera); Lau fra (Launaea fragilis); Lav sto (Lavandula stoechas); Mar sup (Marrubium supinum); Opu fic (Opuntia ficus-indica); Par suf (Paronychia suffruticosa); Phl lic (Phlomis lychnitis); Pip mil (Piptatherum miliaceum); Pla alb (Plantago albicans); Pla gra (Plàntago albicans); Poa lig (Poa ligulata); Pot reu (Potentilla reuteri); Pru pro (Prunus prostrata); Pso bit (Psoralea bituminosa); Ret sph (Retama sphaerocarpa); San ros (Santolina rosmarinifolia); Scl pol (Scleranthus 
polycarpus); Sed sed (Sedum sediforme); Ses can (Sesamoides canescens); Sid lut (Sideritis luteola); Sil lat (Silene latifolia); Sti par (Stipa parviflora); Sti ten (Stipa tenacissima); Teu gra (Teucrium gracillimum); Teu com (Teucrium compactum); Tha vil (Thapsia villosa); Thy hir (Thymelaea hisuta); Thy bae (Thymus baeticus); Thy gad (Thymus gadorensis); Thy gra (Thymus gracillis); Ver gig (Verbascum giganteum);

\section{BIBLIOGRAFÍA}

AGUILAR, J., M. SIMÓN, J. FERNÁNDEZ, I. GARCÍA, J.M. MILÁN y J.F. MOTA -1987Mapa de Suelos de Fiñana (1:100.000). LUCDEME.

AUERBACH, M. \& A. SHMIDA - 1993-Vegetation change along an altitudinal gradient on $\mathrm{Mt}$ Hermon, Israel - no evidence for discrete communities. Journal of Ecology, 81: 25-33.

GHANZANFAR, S.A. -1991- Vegetation structure and phytogeography of Jabal Shams, an arid mountain in Oman. Journal of Biogeography, 18: 299-309.

GRIME, J.P. -1973- Control of species density in herbaceus vegetation. J. Envir. Man., 1: 151167.

GRIME, J.P. - 1979- Plant Strategies and Vegetation Process. New York: Wiley.

KENT, M. \& P. COKER -1992-Vegetation description and analisis: a practical approach. CRC Press.

MONTERO DE BURGOS, J.L. y J.L. GONZÁLEZ REBOLLAR -1983-Diagramas Bioclimáticos. Madrid.

OYONARTE, C. J. PEÑAS, J. CABELLO, \& J.F. MOTA -1995- Climatic Gradient and Vegetational Diversity in the Sierra Gádor (Almería, Spain). In: W.Wildpret, Islands and High Mountain Vegetation: Biodiversity, Bioclimate and Conservation. Serv. Publicaciones Universidad de La Laguna.

PEÑAS, J. F. VALLE, J. CABELLO y J.F. MOTA POVEDA -1995- Los matorrales como discrminantes biogeográficos en la $S^{a}$ de los Filabres (Almería, España). Boletín del Instituto de Estudios Almeriense, Ciencias 13.

RIVAS MARTÍNEZ, S., W. WILDPRET, T.E. DÍAZ, P.L. PÉREZ DE PAZ, M. DEL ARCO, \& O. RODRÍGUEZ, -1993-Excursion guide.
Outline vegetation of Tenerife Island (Canary Islands). Itinera Geobotanica, 7:5-168.

WHITTAKER, R.H. -1960- Vegetation of the Siskiyou Mountains, Oregon and California. Ecol. Monogr., 30:279-338.

WILSON, M.V.\& C.L. MOHLER -1983-Measuring compositional change along gradients. Vegetatio, 54: 129-141.

WILSON, M.V. \& A. SHMIDA, -1984- Measuring Beta Diversity with Presence-Absence Data. Journal of Ecology, 72: 1055-1064.

Aceptado para su publicación en Junio de 1995

Dirección de los autores. J. PEÑAS, J. CABELLO Y J. F. MOTA: Departamento de Biología Vegetal, Producción Vegetal y Ecología. Esc. Politécnica Superior. Universidad de Almería. C. OYONARTE: Dpto. Edafología y Química Agrícola. Universidad de Almería. E-04120 Almería (España). 